QUARTERLY OF APPLIED MATHEMATICS

VOLUME LXX, NUMBER 1

MARCH 2012, PAGES 45-51

S $0033-569 X(2011) 01248-4$

Article electronically published on August 26, 2011

\title{
ESCAPE TO INFINITY IN THE PRESENCE OF MAGNETIC FIELDS
}

BY

A. DÍAZ-CANO (IMI and Departamento de Álgebra, Facultad de Ciencias Matemáticas, Universidad Complutense de Madrid, E-28040 Madrid, Spain)

AND

F. GONZÁLEZ-GASCÓN (Departamento de Física Teórica II, Facultad de Ciencias Físicas, Universidad Complutense de Madrid, E-28040 Madrid, Spain)

Abstract. Escape to infinity is proved to occur when a charge moves under the action of the magnetic field created by a finite number of planar closed wires.

1. Introduction. The late Prof. Ulam, cf. [1], repeatedly stressed the importance of the study of the magnetic field $\mathbf{B}$ created by closed (cyclic or periodic) wires, as a source of mathematical problems: the presence of knotted streamlines of $\mathbf{B}$, ergodic streamlines in open sets of $\mathbb{R}^{3}$, applications in plasma physics and biology, [2]. Recently, [3], the unreachability of the wires (the sources of $\mathbf{B}$ ) when a charged particle moves in $\mathbb{R}^{3}$ under the presence of $\mathbf{B}$ was proved either for a finite number of parallel wires (that is, for a finite number of parallel straight lines) or for a finite number of circular wires, lying on parallel planes $\pi_{i}$, its centers lying on a straight line $L$ orthogonal to the planes $\pi_{i}$.

We study in this paper the escape to infinity of electric charges under the presence of the magnetic field $\mathbf{B}$ created by closed wires traversed by electrical intensities $I_{i}$. It is assumed that the electric charges interact with $\mathbf{B}$ via the Lorentz equation,

$$
\ddot{\mathbf{x}}=\dot{\mathbf{x}} \wedge \mathbf{B} \text {. }
$$

For electric charges, interacting with the electric field $\mathbf{E}$ created by pointlike charges, escape to infinity was studied in $[4-5$. The reader should have a look at references 6 ] and [7, where Matsuno and Goriely and Hyde studied the escape of $\mathbb{R}^{n}$ vector fields via Painlevé analysis. The references in [8]-[22] are also useful concerning the escape for polynomial vector fields.

Received April 30, 2010.

2010 Mathematics Subject Classification. Primary 78A35; Secondary 34A34.

Key words and phrases. Escape to infinity, magnetic field, Lorentz equation.

The first author was partially supported by Spanish GAAR MTM2008-00272 and GAAR Grupos UCM 910444 .

E-mail address: adiaz@mat.ucm.es

E-mail address: fggascon@fis.ucm.es

(C) 2011 Brown University 
Concerning escape to infinity in the presence of magnetic fields $\mathbf{B}(\mathbf{x})$ created or not created by a finite number of cyclic wires, the authors are not aware of previous references studying this problem.

The result obtained here is (see Section 2): if all the cyclic wires lie on a plane $\pi$, then escape to infinity is possible if the initial conditions $\left(\mathbf{x}_{0}, \dot{\mathbf{x}}_{0}\right)$ (see equation (1.1)) satisfy:

$$
\left.\begin{array}{l}
\mathbf{x}_{0} \in \pi \\
\dot{\mathbf{x}}_{0} \| \pi \\
\left\|\mathbf{x}_{0}\right\| \text { large }
\end{array}\right\}
$$

$\|\cdot\|$ being the standard Euclidean norm. This result also holds (see Section 3) in the relativistic case.

A trivial case of escape to infinity appears when the cyclic spires are circular and their centers lie on a straight line $L$ orthogonal to their (parallel) planes. In this case $\left.\mathbf{B}(\mathbf{x})\right|_{L} \| L$ and escape to infinity trivially occurs along $L$ (for $\dot{\mathbf{x}}_{0} \| L$ ).

The results of the present paper could be of interest in the study of plasma physics.

2. Escape under the magnetic field of a dipole. We show in this section that escape to infinity occurs in the equator plane of a dipole (equator of the Earth considered magnetically as a dipole oriented in the North-South direction) and a useful application of this fact is given (see equation (2.11) and the lines following it).

In fact, a magnetic dipole, parallel or antiparallel to the $z$-axis gives rise to a magnetic field $\mathbf{B}(\mathbf{x}), \mathbf{x}=(x, y, z)$, which on the points of the $(x, y)$-plane (equatorial plane of the dipole) is of the form, [23],

$$
\left.\begin{array}{l}
\mathbf{B}=\left(0,0, B_{\perp}\right) \\
B_{\perp}=k\left(x^{2}+y^{2}\right)^{-3 / 2}
\end{array}\right\},
$$

$k$ being a real number proportional to the modulus of the magnetic moment of the dipole.

Under these circumstances, an electric charge (we take its charge and mass equal to one) moving under this vector field $\mathbf{B}$, with initial conditions $\left(x_{0}, y_{0}, 0 ; \dot{x}_{0}, \dot{y}_{0}, 0\right)$ will never, of course, abandon the plane $z=0$.

We now prove that this charge, under suitable initial conditions, can escape to infinity in the plane $z=0$. Indeed, its nonrelativistic equations of motion are:

$$
\left.\begin{array}{l}
\ddot{x}=B_{\perp}(x, y) \dot{y} \\
\ddot{y}=-B_{\perp}(x, y) \dot{x}
\end{array}\right\},
$$

where $B_{\perp}(x, y)$ is given in equation (2.1). Equation (2.2) possesses the pair of first integrals:

$$
\left.\begin{array}{l}
\dot{x}^{2}+\dot{y}^{2} \\
x \dot{y}-y \dot{x}+\frac{1}{2} \int B_{\perp}(u) d u \\
u=x^{2}+y^{2}
\end{array}\right\},
$$


which in polar coordinates $(r, \phi)$ become:

$$
\left.\begin{array}{l}
\dot{r}^{2}+r^{2} \dot{\phi}^{2} \\
r^{2} \dot{\phi}+\frac{1}{2} \int B_{\perp}(u) d u \\
u=r^{2}
\end{array}\right\} .
$$

For a dipole $B_{\perp}(u)=\frac{k}{u^{3 / 2}}$, the term $\frac{1}{2} \int B_{\perp}(u) d u$ in equation (2.4) becomes:

$$
-\frac{k}{r} \text {. }
$$

Calling $v_{0}^{2}$ and $L$ the values of the first integrals in equation (2.4) we get (choosing $\dot{r}>0)$ :

$$
\left.\begin{array}{l}
\dot{\phi}=\left(L+\frac{k}{r}\right) r^{-2} \\
\dot{r}=\sqrt{v_{0}^{2}-\left(L+\frac{k}{r}\right)^{2} r^{-2}}
\end{array}\right\} .
$$

Escape to infinity will occur if the radicand $R:=v_{0}^{2}-\left(L+\frac{k}{r}\right)^{2} r^{-2}$ in $\dot{r}$ never vanishes.

We now set $k>0$. This can always be assumed by choosing appropriately the direction of the $z$-axis. The reader can check that $R$ does not vanish when the following inequality holds:

$$
L^{2}<4 v_{0} k \text {. }
$$

We now write (for further use) the expressions of $r^{\prime}=\frac{d r}{d \phi}, r^{\prime \prime}=\frac{d^{2} r}{d \phi^{2}}$ and $\rho(\phi)(\rho=$ radius of curvature) for the escape orbits $r(\phi)$ of equations (2.2) when $B_{\perp}=k r^{-3}$ (see equation (2.1)):

$$
r^{\prime}=\frac{d r}{d \phi}=\frac{\dot{r}}{\dot{\phi}}=\frac{\left[v_{0}^{2}-\left(L+\frac{k}{r}\right)^{2} r^{-2}\right]^{1 / 2}}{\left(L+\frac{k}{r}\right) r^{-2}}=r^{2} \frac{v_{0}}{L}+O(r),
$$

$O(r)$ standing for a term of type $r A_{1}(r)$, with $A_{1}$ bounded for large values of $r$;

$$
\left.\begin{array}{l}
r^{\prime \prime}=\frac{d r^{\prime}}{d \phi}=2 \frac{v_{0}}{L} r \frac{d r}{d \phi}+\ldots=2 \frac{v_{0}}{L} r \cdot r^{2} \frac{v_{0}}{L}+\ldots=2 \frac{v_{0}^{2}}{L^{2}} r^{3}+O\left(r^{2}\right) \\
O\left(r^{2}\right)=r^{2} A_{2}(r)
\end{array}\right\},
$$

with $A_{2}$ bounded for large values of $r$. The radius of curvature is given by, [24]:

$$
\rho(\phi)=\frac{\left(r^{2}+r^{2}\right)^{3 / 2}}{2 r^{\prime 2}+r^{2}-r r^{\prime \prime}}=\frac{\left(r^{2}+r^{4} \frac{v_{0}^{2}}{L^{2}}\right)^{3 / 2}}{r^{2}}+\ldots=\frac{v_{0}^{3}}{L^{3}} r^{4}+O\left(r^{3}\right) .
$$

We give now an application of the above formulas when the magnetic field $\mathbf{B}$ is of the form

$$
\left.\begin{array}{l}
\mathbf{B}=\left(0,0, B_{\perp}^{*}(x, y)\right) \\
B_{\perp}^{*} \leq \frac{k}{r^{3}} \\
B_{\perp}^{*} \neq 0 \\
r \text { large }
\end{array}\right\}
$$


From equations (2.2) and the intrinsic equations of motion of the charge $q$ (remember $m=1, q=1)$

$$
\frac{d v}{d t}=0, \quad \frac{v^{2}}{\rho^{*}}=v B_{\perp}^{*},
$$

where $v=\left(\dot{x}^{2}+\dot{y}^{2}\right)^{1 / 2}$ and $\rho^{*}$ is the radius of curvature of the trajectory under the magnetic field $B_{\perp}^{*}$. We get (via $B_{\perp}^{*} \leq \frac{k}{r^{3}}$ ):

$$
\rho^{*} \geq \rho_{\text {dipole }}
$$

$\rho_{\text {dipole }}$ being defined by

$$
\frac{v^{2}}{\rho_{\text {dipole }}}=v B_{\perp}=v \frac{k}{r^{3}} .
$$

Let us now prove that $\rho^{*}(r)$ is defined for all values of $r$. In fact, otherwise (and by equation (2.13) $)$ it would blow up for a certain $r_{1} \in \mathbb{R}\left(\lim _{r \rightarrow r_{1}} \rho^{*}=+\infty\right)$ and therefore we would have (and by equation (2.12))

$$
\left.\begin{array}{l}
\left.B_{\perp}^{*}(P)=0\right) \\
P \in\left\{r^{2}=r_{1}^{2}\right\}
\end{array}\right\},
$$

contradicting one of the assumptions of equations (2.11). Therefore $r_{1} \in \mathbb{R}$ cannot exist and $\rho^{*}$ is defined for all values of $r$ (escape to infinity).

Note that in the relativistic case, equations (2.2) become:

$$
\left.\begin{array}{l}
\frac{d}{d t}(\gamma \dot{\mathbf{x}})=B_{\perp}(\dot{y},-\dot{x}) \\
\mathbf{x}=(x, y) \\
\gamma=\left(1-\dot{\mathbf{x}}^{2}\right)^{-1 / 2} \\
c=\text { speed of light }=1, \quad \dot{\mathbf{x}}^{2}<1
\end{array}\right\}
$$

Since $v^{2}=\dot{\mathrm{x}}^{2}=\dot{x}^{2}+\dot{y}^{2}$ is again a first integral, equations (2.16) become

$$
\left.\begin{array}{l}
\ddot{x}=\gamma_{0}^{-1} B_{\perp} \dot{y} \\
\ddot{y}=-\gamma_{0}^{-1} B_{\perp} \dot{x} \\
\gamma_{0}=\left(1-v_{0}^{2}\right)^{-1 / 2} \\
v_{0}^{2}<1
\end{array}\right\} .
$$

We can now include the constant factor $\gamma_{0}^{-1}$ in $B_{\perp}(x, y)$ and proceed as in the first part of this section.

We can therefore conclude that when the following equations hold (see equations (2.11) $)$ :

$$
\left.\begin{array}{l}
\gamma_{0}^{-1} B_{\perp}^{*} \leq \frac{k}{r^{3}} \\
B_{\perp}^{*} \neq 0 \\
r \text { large }
\end{array}\right\}
$$

and relativistic escape to infinity is possible as well. 
3. Escape to infinity in the magnetic field of closed, planar wires. We prove in this section that for a finite number $N$ of plane closed wires lying on the $(x, y)$-plane there is escape to infinity. Note that in this case $B_{\perp}$ is not necessarily symmetric under rotations around the $z$-axis.

In fact, from the Biot-Savart formula we get

$$
\left.\begin{array}{l}
\mathbf{B}(x, y, 0)=\sum_{i=1}^{N} I_{i} \int_{0}^{2 \pi} \frac{\left(\mathbf{x}-\mathbf{x}_{i}(t)\right)}{\left\|\mathbf{x}-\mathbf{x}_{i}(t)\right\|^{3}} \wedge \dot{\mathbf{x}}_{i}(t) d t \\
\mathbf{x}=(x, y, 0), \mathbf{x}_{i}(t)=\left(x_{i}(t), y_{i}(t), 0\right)
\end{array}\right\},
$$

and

$$
B_{\perp}(x, y, 0)=\sum_{i=1}^{N} I_{i} \int_{0}^{2 \pi} \frac{\left(x-x_{i}\right) \dot{y}_{i}-\left(y-y_{i}\right) \dot{x}_{i}}{\left\|\mathbf{x}-\mathbf{x}_{i}(t)\right\|^{3}} d t
$$

$I_{i} \in \mathbb{R}$ being the intensities on the closed wires and $\mathbf{x}_{i}(t)$ a parametrization of them.

We now write $\left\|\mathbf{x}-\mathbf{x}_{i}(t)\right\|^{3}$ in the form:

$$
\left.\begin{array}{l}
\left\|\mathbf{x}-\mathbf{x}_{i}(t)\right\|^{3}=\left(\left(x-x_{i}(t)\right)^{2}+\left(y-y_{i}(t)\right)^{2}+z_{i}(t)^{2}\right)^{3 / 2} \\
=\left(x^{2}+y^{2}-2 x x_{i}-2 y y_{i}+\mathbf{x}_{i}(t)^{2}\right)^{3 / 2}=r^{3 / 2}\left(1+\frac{-2 x x_{i}-2 y y_{i}+\mathbf{x}_{i}(t)^{2}}{r^{2}}\right)^{3 / 2}, \\
x^{2}+y^{2}=r^{2}
\end{array}\right\} .
$$

On substitution of (3.3) into (3.2) and expansion of $\left(1+\frac{-2 x x_{i}-2 y y_{i}+\mathbf{x}_{i}(t)^{2}}{r^{2}}\right)^{3 / 2}$ in powers of $\frac{1}{r^{2}}$ (remember that $\frac{-2 x x_{i}-2 y y_{i}+\mathbf{x}_{i}(t)^{2}}{r^{2}} \rightarrow 0$ when $r \rightarrow \infty$ ) we get:

$$
\left.\begin{array}{l}
B_{\perp}(x, y, 0)=\frac{k^{\prime}}{r^{3}}+\frac{1}{r^{4}} A(r, \phi) \\
k^{\prime} \in \mathbb{R}
\end{array}\right\},
$$

$k^{\prime}$ being defined by

$$
k^{\prime}=\sum_{i=1}^{N} I_{i} \int \frac{-x_{i} \dot{y}_{i}+y_{i} \dot{x}_{i}}{\left\|\mathbf{x}-\mathbf{x}_{i}(t)\right\|^{3}} d t=\sum I_{i} A_{i},
$$

and $A_{i}$ being the areas of the closed wires $\mathcal{C}_{i}$ :

$$
A_{i}=\int_{\mathcal{C}_{i}}\left(-x_{i} \dot{y}_{i}+y_{i} \dot{x}_{i}\right) d t .
$$

On the other hand, the term $A(r, \phi)$ in (3.4) is bounded for large values of $r$.

We now assume that $k^{\prime}>0$ (choosing the direction of the $z$-axis conveniently) or $k^{\prime}=0$.

When $k^{\prime}>0$, from equation (3.4) we get

$$
\left.\begin{array}{l}
B_{\perp}(x, y, 0) \leq \frac{2 k^{\prime}}{r^{3}} \\
r \text { large }
\end{array}\right\} .
$$

Therefore, applying the reasoning of Section 2 (see the lines following equation (2.13)), escape to infinity has been proved. Note that by equation (3.4), $B_{\perp}$ cannot vanish for 
large values of $r$. Note also that $k^{\prime}$ cannot vanish unless $I_{i}$ is positive for some wires and negative for other ones.

Escape to infinity also occurs when $k^{\prime}=0$ since equation (3.4) becomes in this case:

$$
\left.\begin{array}{l}
B_{\perp}(x, y, 0)=\frac{L(\phi)}{r^{n^{\prime}}}+\frac{A_{1}(r, \phi)}{r^{n^{\prime}+1}} \\
n^{\prime}>3
\end{array}\right\},
$$

where $L(\phi)$ does not vanish identically and is positive (via a convenient choosing of the direction of the $z$-axis) and $\left.A_{(} r, \phi\right)$ is bounded for $r$ large.

On the other hand, equation (3.7) now becomes:

$$
\left.\begin{array}{l}
B_{\perp}(x, y, 0) \leq \frac{2 \operatorname{Max}(L(\phi))}{r^{n^{\prime}}} \\
r=\text { large }
\end{array}\right\},
$$

and the existence of escape trajectories follows by the reasoning following equation (3.7).

4. Open problems. In the case of a vector field $\mathbf{B}$ created by a finite set of nonplanar closed wires, some of which could be knotted, the possibility of escape to infinity is still open. In fact, it would be nice to prove that escape to infinity is possible (or is not possible) when $\mathbf{B}(\mathbf{x})$ is created by a particular closed, but nonplanar, wire.

\section{REFERENCES}

[1] S. Ulam, Problems in modern mathematics, Science Editions, John Wiley \& Sons, Inc., 1964. MR0280310 (43:6031)

[2] J. Sarvas, Basic mathematical and electromagnetic concepts of the biomagnetic inverse problem, Phys. Med. Biol. 32 (1987), 11-22.

[3] F. González-Gascón, D. Peralta-Salas, Motion of a charge in the magnetic field created by wires: impossibility of reaching the wires, Phys. Lett. A. 333 (2004), 72-78. MR2101963 (2005g:78009)

[4] F. González-Gascón, D. Peralta-Salas, Escape to infinity in a Newtonian potential, J. Phys. A 33 (2000), 5361-5368. MR1783734 (2001j:70009)

[5] F. González-Gascón, D. Peralta-Salas, Escape to infinity under the action of a potential and a constant electromagnetic field, J. Phys. A 36 (2003), 6441-6455. MR.1987441(2004f:70042)

[6] Y. Matsuno, Two-dimensional dynamical system associated with Abel's nonlinear differential equation, J. Math. Phys. 33 (1992), 412-421. MR.1141541 (92h:34025)

[7] A. Goriely, C. Hyde, Finite-time blow-up in dynamical systems, Phys. Lett. A 250 (1998), 311-318. MR 1666111 (2000e:34056)

[8] C. Marchioro, Solution of a three-body scattering problem in one dimension, J. Math. Phys. 11 (1970), 2193-2196.

[9] L.P. Fulcher, B.F. Davis, D.A. Rowe, An approximate method for classical scattering problems, Amer. J. Phys. 44 (1976), 956-959.

[10] L. Vaserstein, On systems of particles with finite-range and/or repulsive interactions, Commun. Math. Phys. 69 (1979), 31-56. MR547525 (81b:58028)

[11] G. Galperin, Asymptotic behaviour of particle motion under repulsive forces, Commun. Math. Phys. 84 (1982), 547-556. MR667760 (83m:70023)

[12] E. Gutkin, Integrable Hamiltonians with exponential potential, Phys. D 16 (1985), 398-404. MR 805712 (86k:58036)

[13] E. Gutkin, Asymptotics of trajectories for cone potentials, Phys. D 17 (1985), 235-242. MR815287 (87c:58040)

[14] V.J. Menon, D.C. Agrawal, Solar escape revisited, Amer. J. Phys. 54 (1986), 752-753.

[15] E. Gutkin, Continuity of scattering data for particles on the line with directed repulsive interactions, J. Math. Phys. 28 (1987), 351-359. MR872012 (88f:70011) 
[16] A. Hubacher, Classical scattering theory in one dimension, Comm. Math. Phys. 123 (1989), 353375. MR.1003425 (90f:58062)

[17] V. Moauro, P. Negrini, W.M. Oliva, Analytic integrability for a class of cone potential Hamiltonian systems, J. Differential Equations 90 (1991), 61-70. MR.1094449 (92b:58072)

[18] G. Fusco, W.M. Oliva, Integrability of a system of $N$ electrons subjected to Coulombian interactions, J. Differential Equations 135 (1997), 16-40. MR1434913 (98c:70012)

[19] C. Coleman, Boundedness and unboundedness in polynomial differential systems, Nonlinear Anal. 8 (1984), 1287-1294. MR764913 (86b:34064)

[20] Z.F. Zhang, T.R. Ding, W.Z. Huang, Z.X. Dong, Qualitative theory of differential equations, Translations of Mathematical Monographs 101, American Mathematical Society, 1992. MR1175631 (93h:34002)

[21] H. Röhrl, S. Walcher, Projections of polynomial vector fields and the Poincaré sphere, J. Differential Equations 139 (1997), 22-40. MR.1467351 (98j:34050)

[22] A. García, E. Pérez-Chavela, A. Susin, A generalization of the Poincaré compactification, Arch. Ration. Mech. Anal. 179 (2006), 285-302. MR2209132 (2007f:37026)

[23] G. Arfken, Mathematical methods for physicists, Academic Press, 1966. MR0205512 (34:5339)

[24] M.P. do Carmo, Differential Geometry of curves and surfaces, Prentice-Hall, 1976. MR0394451 $(52: 15253)$ 